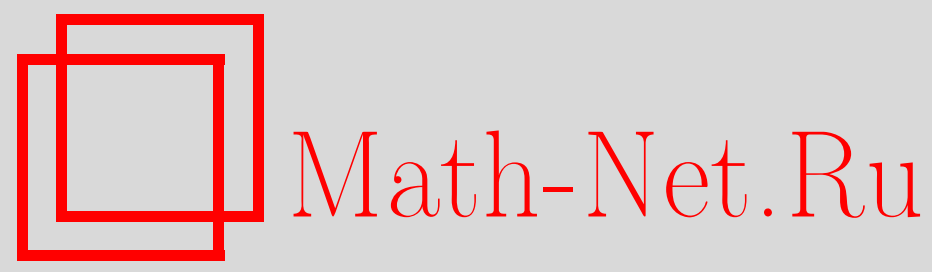

Н. Н. Токарева, Группа автоморфизмов множества бентфункций, Дискрет. матем., 2010, том 22, выпуск 4, 34-42

DOI: https://doi.org/10.4213/dm1117

Использование Общероссийского математического портала Math-Net.Ru подразумевает, что вы прочитали и согласны с пользовательским соглашением http://www.mathnet.ru/rus/agreement

Параметры загрузки:

IP: 54.157 .27 .8

26 апреля 2023 г., 12:32:06 
УДК 519.7

\title{
Группа автоморфизмов множества бент-функций
}

\author{
() 2010 г. Н. Н. Токарева
}

\begin{abstract}
Бент-функции - это булевы функции от четного числа переменных, удаленные от множества всех аффинных функций на максимально возможное расстояние. В работе показано, что каждое изометричное отображение множества булевых функций от $n$ переменных в себя, оставляющее класс бент-функций на месте, является комбинацией аффинного преобразования координат и сдвига на аффинную функцию. Доказано, что аффинные функции - это в точности все те булевы функции, которые удалены от класса бент-функций на максимально возможное расстояние.

Работа выполнена при поддержке гранта Президента Российской Федерации для молодых российских ученых, грант МК 1250.2009.1, Российского фонда фундаментальных исследований, проекты 08-01-00671, 09-01-00528, 10-01-00424, и ФЦП «Научные и научно-педагогические кадры инновационной России» на 2009-2013 гг., государственный контракт 02.740.11.0429.
\end{abstract}

\section{1. Введение}

Бент-функции - это булевы функции от четного числа переменных, удаленные от множества всех аффинных функций на максимально возможное расстояние. Они были введены О. Ротхаусом [6]. В настоящее время бент-функции активно изучаются и имеют много приложений в теории кодирования, криптографии, цифровой сотовой связи и других областях (см. обзоры $[3,4])$. Тем не менее, в теории бент-функций много открытых вопросов. Среди них - вопрос о группе автоморфизмов множества бент-функций, ответ на который дается в данной работе.

Пусть $A-$ невырожденная двоичная $n \times n$ матрица, пусть $b$ и $c-$ двоичные векторы длины $n$, и $d-$ константа. Известно, что любое отображение вида

$$
g(x) \rightarrow g(A x+b)+\langle c, x\rangle+d,
$$

заданное на множестве булевых функций от $n$ переменных, оставляет класс бент-функций на месте. Существуют ли другие изометричные отображения множества булевых функций в себя, оставляющие неподвижным класс бент-функций? В данной работе показано, что других таких отображений нет.

Рассмотрим структуру статьи. Основная часть статьи посвящена доказательству того, что для любой неаффинной функции $f$ найдется такая бент-функция $g$, что функция $f+g$ не является бент-функцией (теорема 1). Другими словами, множество бент-функций замкнуто относительно прибавления только аффинных булевых функций. Из этого факта будет следовать, что аффинные функции - это в точности все те булевы функции, 
которые удалены от класса бент-функций на максимально возможное расстояние. Другими словами, существует в некотором смысле дуальность между определениями бентфункций и аффинных функций. Далее устанавливается, что множество бент-функций и множество аффинных функций имеют одинаковые группы автоморфизмов. Этой общей группой является полупрямое произведение полной аффинной группы $G A(n)$ на $\mathbf{Z}_{2}^{n+1}$ (см. теорему 2).

\section{2. Определения и факты}

Пусть $\langle x, y\rangle$ - обычное скалярное произведение двоичных векторов $x, y \in \mathbf{Z}_{2}^{n}$ по модулю 2. Расстоянием Хэмминга $d(x, y)$ между векторами $x$ и $y$ называется число координат, в которых они различаются.

Под расстоянием $\operatorname{dist}(f, g)$ между булевыми функциями $f$ и $g$ будем понимать расстояние между их векторами значений. Через $\operatorname{supp}(f)$ обозначим носитель функции $f$, то есть множество тех векторов, на которых $f$ равна единице. Известно, что каждая булева функция $f$ может быть представлена в алгебраической нормальной форме, степень которой обозначим через $\operatorname{deg}(f)$. Булевы функции степени 1 называются аффинными и имеют вид $\langle c, x\rangle+d$ для подходящего вектора $c$ и константы $d$. Множество всех аффинных функций от $n$ переменных обозначим через $\mathscr{A}_{n}$.

Преобразованием Уолша-Адамара булевой функции $f$ от $n$ переменных называется целочисленная функция

$$
W_{f}(y)=\sum_{x}(-1)^{\langle x, y\rangle+f(x)} .
$$

Бент-функцией называется булева функция от четного числа $n$ переменных такая, что модуль каждого коэффициента Уолша-Адамара $W_{f}(y)$ этой функции равен $2^{n / 2}$.

Производной булевой функции $f$ по направлению $y$ называется бинарная сумма $f(x)+f(x+y)$. Заметим, что булева функция $f$ аффинна тогда и только тогда, когда ее производная по любому направлению является константой.

Эквивалентно, бент-функции могут быть определены (см. [1]) следующим образом.

Предложение 1. Булева функция g является бент-функиией, тогда и только тогда, когда ее производная по любому ненулевому направлению у уравновешена, то есть выполняется равенство

$$
\sum_{x}(-1)^{g(x)+g(x+y)}=0 .
$$

Множество всех бент-функций от $n$ переменных обозначим через $\mathscr{P}_{n}$. Хорошо известно следующее свойство бент-функций.

Предложение 2. Пусть $A-$ невырожденная двоичная $n \times n$ матрица, $b$ и $c$ - двоичные векторы, $d-$-константа. Любое отображение вида

$$
g(x) \rightarrow g(A x+b)+\langle c, x\rangle+d,
$$

заданное на множестве булевых функций от $n$ переменных, оставляет класс бентфункиий на месте.

Далее нам потребуется известная конструкция бент-функций Мак-Фарланда [5]. 
Предложение 3. Функичия

$$
f\left(x^{\prime}, x^{\prime \prime}\right)=\left\langle x^{\prime}, \pi\left(x^{\prime \prime}\right)\right\rangle+h\left(x^{\prime \prime}\right)
$$

является бент-функцией от $n$ переменных, где $\pi$-любая перестановка на $\mathbf{Z}_{2}^{n / 2}$ и булева функция $h$ от $n / 2$ переменных произвольна.

Заметим, что разбиение переменных на две равные части $x^{\prime}$ и $x^{\prime \prime}$ может быть любым.

Рассмотрим векторы, у которых фиксированы значения некоторых $n-k$ координат, а значения остальных координат выбираются произвольно. Множество всех таких векторов называется гранью размерности $k$ пространства $\mathbf{Z}_{2}^{n}$. Например, множество

$$
\Gamma=\left\{\left(x^{\prime}, x^{\prime \prime}\right): x^{\prime \prime}=a\right\}
$$

является гранью размерности $n / 2$, где $x^{\prime}, x^{\prime \prime} \in \mathbf{Z}_{2}^{n / 2}$.

\section{3. О сдвигах класса бент-функций}

Докажем следующий факт, из которого в качестве следствий и будут получены основные результаты работы.

Теорема 1. Для любой неаффинной функции $f$ найдется такая бент-функция $g$, что функция $f+g$ не является бент-функцией.

Доказательство. Предположим, что для некоторой фиксированной функции $f$ такой, что $\operatorname{deg}(f) \geqslant 2$, справедливо равенство $f+\mathscr{B}_{n}=\mathscr{B}_{n}$. Покажем, что это приведет к противоречию.

Идея доказательства состоит в следующем. Сначала покажем, что некоторая сумма должна быть равна нулю для любой бент-функции (см. далее сумму (1)). Затем в классе Мак-Фарланда найдем бент-функцию - контрпример $g^{\prime}$, для которой это равенство не будет выполняться. Бент-функция $g^{\prime}$ будет получена из специально выбранной бентфункции $g$ инвертированием ее значений на некоторой грани $\Gamma$ размерности $n / 2$. Ключевым условием для возможности выбора такой грани является то, что для некоторого ненулевого $y$ множество $D=\operatorname{supp}(f(x)+f(x+y))$ будет собственным подмножеством пространства $\mathbf{Z}_{2}^{n}$, что возможно тогда и только тогда, когда $f$ неаффинна.

Доказательство удобно разделить на несколько этапов.

\section{1. Равенство для любой бент-функции}

Поскольку $\operatorname{deg}(f) \geqslant 2$, найдется такой ненулевой вектор $y$, что производная функции $f$ по направлению $y$ не является константой. Можно считать, что $y=1$. Действительно, так как если $y$ - другой ненулевой вектор, то от функции $f$ перейдем к функции $f^{\prime}(x)=f(A x)$, где $A \cdot 1=y$. Очевидно, что производная функции $f^{\prime}$ по направлению 1 не является константой, и так же как и для $f$, выполняется равенство $f^{\prime}+\mathscr{B}_{n}=\mathscr{B}_{n}$ (то есть можно доказывать теорему для $f^{\prime}$ ). Поэтому всюду далее считаем, что $y=1$.

Пусть $g-$ произвольная бент-функция. Тогда $f+g$ - также бент-функция, и согласно утверждению 1 выполняются равенства

$$
\begin{aligned}
\sum_{x}(-1)^{g(x)+g(x+y)} & =0, \\
\sum_{x}(-1)^{g(x)+f(x)+g(x+y)+f(x+y)} & =0 .
\end{aligned}
$$


Вычитая из первого равенства второе, получаем, что

$$
\sum_{x}(-1)^{g(x)+g(x+y)}\left(1-(-1)^{f(x)+f(x+y)}\right)=0 .
$$

Обозначим через $D$ множество $\operatorname{supp}(f(x)+f(x+y))$. Тогда для любой бент-функции $g$ должно выполняться равенство

$$
\sum_{x \in D}(-1)^{g(x)+g(x+y)}=0 .
$$

\section{2. Выбор грани}

Так как функция $f(x)+f(x+y)$ не является константой, множество $D$ не пусто и не совпадает со всем булевым кубом. Тогда существует $(n / 2)$-мерная грань $\Gamma$ такая, что она имеет непустое пересечение и с множеством $D$, и с его дополнением $\mathbf{Z}_{2}^{n} \backslash D$, то есть выполняются неравенства

$$
0<m<|\Gamma|=2^{n / 2},
$$

где $m=|\Gamma \cap D|$. Действительно, такую грань всегда можно построить, например, как грань через любой вектор $u \notin D$ и один из векторов $v$ или $v+y$, где $v \in D$, так как либо расстояние $d(u, v)$, либо расстояние $d(u, v+y)$ окажется не превосходящим $n / 2$.

Заметим, что грань $\Gamma+y$ также имеет пересечение мощности $m$ с множеством $D$, поскольку, как нетрудно заметить, $D+y=D$. Отметим также, что грани $\Gamma$ и $\Gamma+y$ не пересекаются (в силу выбора $y=1$ ). Имеет место следующее разбиение множества $D$ :

$$
D=(\Gamma \cap D) \cup((\Gamma+y) \cap D) \cup(D \backslash(\Gamma \cup(\Gamma+y))),
$$

которое потребуется нам в дальнейшем.

Без ограничения общности считаем, что грань Г имеет вид

$$
\Gamma=\left\{\left(x^{\prime}, x^{\prime \prime}\right): x^{\prime \prime}=a\right\}
$$

для некоторого вектора $a \in \mathbf{Z}_{2}^{n / 2}$. В случае, если фиксированные координаты грани расположены иначе, доказательство проводится аналогично.

Пусть множество $\Gamma \cap D$ представляется в виде

$$
\Gamma \cap D=\left\{\left(b^{(1)}, a\right),\left(b^{(2)}, a\right), \ldots,\left(b^{(m)}, a\right)\right\}
$$

для подходящих векторов $b^{(1)}, b^{(2)}, \ldots, b^{(m)}$ длины $n / 2$.

\section{3. Специальное подмножество бент-функций}

Рассмотрим подмножество $G$ бент-функций вида

$$
g\left(x^{\prime}, x^{\prime \prime}\right)=\left\langle x^{\prime}, \pi\left(x^{\prime \prime}\right)\right\rangle
$$

из класса Мак-Фарланда таких, что перестановки $\pi$ являются линейными преобразованиями пространства, то есть каждая перестановка $\pi$ определяется как $\pi\left(x^{\prime \prime}\right)=A x^{\prime \prime}$, 
для подходящей невырожденной матрицы $A$. Покажем, что в классе $G$ найдется такая бент-функция $g$, что сумма

$$
S=\sum_{x \in \Gamma \cap D}(-1)^{g(x)+g(x+y)}
$$

будет не равна нулю. Действительно, распишем эту сумму, подставляя вид произвольной функции из $G$. Поскольку

$$
\pi\left(x^{\prime \prime}+y^{\prime \prime}\right)=A\left(x^{\prime \prime}+y^{\prime \prime}\right)=A x^{\prime \prime}+A y^{\prime \prime},
$$

где $y=\left(y^{\prime}, y^{\prime \prime}\right)$, справедливо равенство

$$
\begin{aligned}
g(x)+g(x+y) & =\left\langle x^{\prime}, A x^{\prime \prime}\right\rangle+\left\langle x^{\prime}+y^{\prime}, A x^{\prime \prime}+A y^{\prime \prime}\right\rangle \\
& =\left\langle x^{\prime}, A y^{\prime \prime}\right\rangle+\left\langle y^{\prime}, A x^{\prime \prime}+A y^{\prime \prime}\right\rangle .
\end{aligned}
$$

Тогда, подставляя в сумму $S$, получаем равенство

$$
S=(-1)^{\gamma} \sum_{i=1}^{m}(-1)^{\left\langle b^{(i)}, A y^{\prime \prime}\right\rangle},
$$

где $\gamma=\left\langle y^{\prime}, A a+A y^{\prime \prime}\right\rangle$ - константа, зависящая от конкретного выбора матрицы $A$. Вектор $y^{\prime \prime}$ ненулевой (по нашему выбору $y^{\prime \prime}=1$ ) и матрица $A$ может быть любой невырожденной матрицей, поэтому вектор $z=A y^{\prime \prime}$ также может быть произвольным ненулевым вектором длины $n / 2$. Таким образом, наша задача показать, что найдется такой ненулевой вектор $z$, что не равна нулю сумма

$$
\sum_{i=1}^{m}(-1)^{\left\langle b^{(i)}, z\right\rangle} .
$$

\section{4. Поиск вектора $z$}

Предположим обратное. Пусть для каждого ненулевого вектора $z$ сумма (4) равна нулю. Рассмотрим двоичную матрицу $M$ размера $(n / 2) \times m$, столбцами которой являются все векторы $b^{(1)}, \ldots, b^{(m)}$. Тогда сумма (4) есть не что иное как разность между числом нулей и числом единиц в линейной комбинации строк матрицы $M$, определяемой вектором $z$ (строка $i$ входит в комбинацию, если $z_{i}=1$ ). По предположению, матрица $M$ должна удовлетворять следующему условию: любая ненулевая линейная комбинация строк матрицы $M$ содержит одинаковое число нулей и единиц. Несложно понять, что, с точностью до перестановки столбцов, эта матрица должна иметь вид

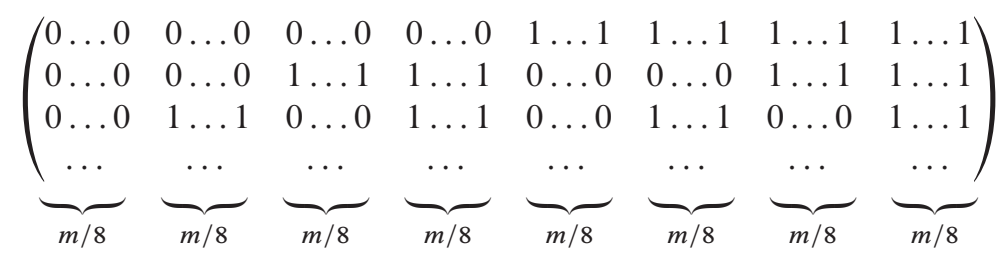

Отсюда сразу следует, что число столбцов матрицы должно быть не меньше, чем $2^{n / 2}$, где $n / 2$ - число строк. Таким образом, для существования такой матрицы $M$ необходимо, чтобы выполнялось неравенство $m \geqslant 2^{n / 2}$. Но это противоречит условию выбора грани $\Gamma$, а именно, неравенству (2). Следовательно, всегда найдется вектор $z$ такой, что сумма (4) не равна нулю. Зафиксируем этот вектор $z$. 


\section{5. Построение функции-контрпримера}

Пусть $A$ - невырожденная матрица такая, что $A y^{\prime \prime}=z$, пусть перестановка $\pi$ определяется равенством $\pi\left(x^{\prime \prime}\right)=A x^{\prime \prime}$. Из условия выбора вектора $z$ следует, что для функции $g\left(x^{\prime}, x^{\prime \prime}\right)=\left\langle x^{\prime}, \pi\left(x^{\prime \prime}\right)\right\rangle$ справедливо равенство

$$
S=\sum_{x \in \Gamma \cap D}(-1)^{g(x)+g(x+y)} \neq 0 .
$$

Определим новую бент-функцию $g^{\prime}$, отличающуюся от $g$ лишь на грани Г. Далее мы увидим, что функции $f+g$ и $f+g^{\prime}$ не могут одновременно быть бент-функциями, что и приведет к противоречию с основным предположением. Итак, пусть

$$
g^{\prime}\left(x^{\prime}, x^{\prime \prime}\right)=g\left(x^{\prime}, x^{\prime \prime}\right)+h\left(x^{\prime \prime}\right),
$$

где

$$
h\left(x^{\prime \prime}\right)= \begin{cases}1, & \text { если } x^{\prime \prime}=a ; \\ 0 & \text { в противном случае. }\end{cases}
$$

Другими словами, $g^{\prime}$ получена из функции $g$ инвертированием ее значений на грани Г. Так как $g$ из класса Мак-Фарланда, функция $g^{\prime}$ - тоже бент-функция. Заметим, что тогда в силу разбиения (3)

$$
\begin{aligned}
\sum_{x \in D}(-1)^{g^{\prime}(x)+g^{\prime}(x+y)}= & \left(\sum_{x \in \Gamma \cap D}(-1)^{g(x)+1+g(x+y)+0)}\right) \\
& +\left(\sum_{x \in(\Gamma+y) \cap D}(-1)^{g(x)+0+g(x+y)+1)}\right. \\
& +\left(\sum_{x \in(D \backslash(\Gamma \cup(\Gamma+y))}(-1)^{g(x)+g(x+y))}\right) \\
= & \left(\sum_{x \in(D \backslash(\Gamma \cup \Gamma+y))}(-1)^{g(x)+g(x+y))}\right)-2 S .
\end{aligned}
$$

Таким образом,

$$
\sum_{x \in D}(-1)^{g^{\prime}(x)+g^{\prime}(x+y)}=\sum_{x \in D}(-1)^{g(x)+g(x+y)}-4 S .
$$

Отсюда из равенства (1) и неравенства (5) следует, что

$$
\sum_{x \in D}(-1)^{g^{\prime}(x)+g^{\prime}(x+y)} \neq 0 .
$$

Но равенство (1) должно выполняться для любой бент-функции, в том числе и для $g^{\prime}$. Полученное противоречие доказывает теорему. 


\section{4. Дуальность определений бент-функций и аффинных функций}

При четном $n$ класс бент-функций $\mathscr{B}_{n}$ можно определить как множество функций, отстоящих от класса всех аффинных булевых функций $\mathscr{A}_{n}$ на максимально возможное расстояние $N_{\max }$, то есть

$$
\mathscr{B}_{n}=\left\{g: \operatorname{dist}\left(g, \mathscr{A}_{n}\right)=N_{\max }\right\} .
$$

При этом известно, что

$$
N_{\max }=2^{n-1}-2^{(n / 2)-1} .
$$

Но можно ли обратить это определение? Другими словами, верно ли, что $\mathscr{A}_{n}-$ это множество всех булевых функций, отстоящих от класса $\mathscr{B}_{n}$ на максимально возможное расстояние $N_{\max }^{\prime}$ ? Пусть

$$
\mathscr{A}_{n}^{\prime}=\left\{f: \operatorname{dist}\left(f, \mathscr{B}_{n}\right)=N_{\text {max }}^{\prime}\right\} .
$$

Покажем, что подобное обращение определений действительно возможно, то есть справедливы равенства

$$
N_{\max }=N_{\max }^{\prime}, \quad \mathscr{A}_{n}=\mathscr{A}_{n}^{\prime} .
$$

Предложение 4. Справедливо равенство

$$
N_{\max }^{\prime}=2^{n-1}-2^{(n / 2)-1} .
$$

Доказательство. По определению

$$
N_{\max }^{\prime}=\max _{f} \min _{g \in \mathscr{P}_{n}} \operatorname{dist}(f, g) .
$$

Заметим, что

$$
\operatorname{dist}(f, g)=2^{n-1}-\frac{1}{2} W_{f+g}(0) .
$$

Поэтому

$$
N_{\max }^{\prime}=2^{n-1}-\frac{1}{2} \min _{f} \max _{g \in \mathscr{P}_{n}}\left|W_{f+g}(0)\right| .
$$

Зафиксируем любую бент-функцию $g^{\prime}$ от $n$ переменных. Поскольку класс $\mathscr{B}_{n}$ замкнут относительно добавления аффинных функций, каждая функция вида $g^{\prime}+l_{a}$, где $l_{a}(x)=\langle a, x\rangle$, является бент-функцией. Заметим, что

$$
W_{f+g^{\prime}+l_{a}}(0)=W_{f+g^{\prime}}(a) .
$$

Тогда очевидно, что

$$
\max _{g \in \Re_{n}}\left|W_{f+g}(0)\right| \geqslant \max _{g=g^{\prime}+l_{a} \text { для некоторого } a}\left|W_{f+g}(0)\right|=\max _{a}\left|W_{f+g^{\prime}}(a)\right| .
$$

Но из равенства Парсеваля для булевой функции $f+g^{\prime}$ следует, что

$$
\max _{a}\left|W_{f+g^{\prime}}(a)\right| \geqslant 2^{n / 2} \text {. }
$$

Отсюда получаем неравенство

$$
N_{\max }^{\prime} \leqslant 2^{n-1}-2^{(n / 2)-1} .
$$

С другой стороны, расстояние $2^{n-1}-2^{(n / 2)-1}$ до класса бент-функций достигается, например, для любой аффинной функции $f$. Утверждение доказано. 
Предложение 5. Справедливо равенство $\mathscr{A}_{n}=\mathscr{A}_{n}^{\prime}$.

Доказательство. Очевидно, что $\mathscr{A}_{n} \subseteq \mathscr{A}_{n}^{\prime}$. Предположим, что существует функция $f \in \mathscr{A}_{n}^{\prime} \backslash \mathscr{A}_{n}$. Тогда по теореме 1 найдется бент-функция $g$ такая, что $f+g$ не является бент-функцией, то есть существует вектор $a$ такой, что

$$
\left|W_{f+g}(a)\right|>2^{n / 2} \text {. }
$$

Рассмотрим бент-функцию

$$
g^{\prime}(x)=g(x)+\langle a, x\rangle .
$$

Для нее справедливо равенство $W_{f+g^{\prime}}(0)=W_{f+g}(a)$ и в силу равенства

$$
\operatorname{dist}\left(f, \mathscr{P}_{n}\right)=2^{n-1}-\frac{1}{2} \max _{g \in \mathscr{P}_{n}}\left|W_{f+g}(0)\right|
$$

заключаем, что $\operatorname{dist}\left(f, \mathscr{B}_{n}\right)<N_{\max }^{\prime}$, что противоречит выбору $f$. Таким образом, $\mathscr{A}_{n}=\mathscr{A}_{n}^{\prime}$.

Заметим, что ключевым фактом, позволившим установить дуальность между определениями аффинных функций и бент-функций, является теорема 1.

\section{5. Автоморфизмы бент-функций}

Отображение $\varphi$ множества всех булевых функций от $n$ переменных в себя называется изометричным, если оно сохраняет расстояния между булевыми функциями, то есть

$$
\operatorname{dist}(\varphi(f), \varphi(g))=\operatorname{dist}(f, g) .
$$

Известно, что любое такое отображение однозначно представляется в виде

$$
g(x) \rightarrow g(s(x))+f(x),
$$

где $s: \mathbf{Z}_{2}^{n} \rightarrow \mathbf{Z}_{2}^{n}-$ любая подстановка, $f-$ произвольная функция от $n$ переменных.

Группой автоморфизмов подмножества булевых функций $M$ называется группа изометричных отображений множества всех булевых функций в себя, оставляющих неподвижным множество $\mathcal{M}$. Обозначим эту группу через Aut $(M)$.

Напомним, что полная аффинная группа $G A(n)$ состоит из всех отображений вида $g(x) \rightarrow g(A x+b)$, где $A-$ невырожденная матрица, $b-$ произвольный вектор. Справедливо следующее утверждение.

Предложение 6. Группа $\operatorname{Aut}\left(\mathscr{A}_{n}\right)$ равна полупрямому произведению полной аффинной группье $G A(n)$ на $\mathbf{Z}_{2}^{n+1}$.

Действительно, для любого автоморфизма (6) сдвиг на функцию $f$ может определяться только аффинной функцией (так как образ нулевой функции - также аффинная функция). Множество всех аффинных функций от $n$ переменных образует группу, изоморфную $\mathbf{Z}_{2}^{n+1}$. Остается отметить, что каждая перестановка $s$, как известно, должна принадлежать группе $G A(n)$ (см., например, [2]).

Теорема 2. Справедливы равенства

$$
\operatorname{Aut}\left(\mathscr{B}_{n}\right)=\operatorname{Aut}\left(\mathscr{A}_{n}\right)=G A(n) \ltimes \mathbf{Z}_{2}^{n+1} .
$$


Доказательство. Очевидно, что $\operatorname{Aut}\left(\mathscr{A}_{n}\right) \subseteq \operatorname{Aut}\left(\mathscr{B}_{n}\right)$. Действительно, для любого отображения $\varphi \in \operatorname{Aut}\left(\mathscr{A}_{n}\right)$ и любой бент-функции $g$

$$
\operatorname{dist}\left(g, \mathscr{A}_{n}\right)=\operatorname{dist}\left(\varphi(g), \varphi\left(\mathscr{A}_{n}\right)\right)=\operatorname{dist}\left(\varphi(g), \mathscr{A}_{n}\right) .
$$

Следовательно, любая бент-функция под действием $\varphi$ переходит в некоторую другую бент-функцию.

Аналогично, $\operatorname{Aut}\left(\mathscr{B}_{n}\right) \subseteq \operatorname{Aut}\left(\mathscr{A}_{n}\right)$. Именно для любого автоморфизма $\psi \in \operatorname{Aut}\left(\mathscr{B}_{n}\right)$ и любой аффинной функции $f$

$$
\operatorname{dist}\left(f, \Re_{n}\right)=\operatorname{dist}\left(\psi(f), \psi\left(\Re_{n}\right)\right)=\operatorname{dist}\left(\psi(f), \Re_{n}\right) .
$$

Отсюда, согласно утверждениям 4 и 5 , следует, что $\psi\left(\mathscr{A}_{n}\right)=\mathscr{A}_{n}$. Получаем, что $\operatorname{Aut}\left(\mathscr{B}_{n}\right)=\operatorname{Aut}\left(\mathscr{A}_{n}\right)$. Из предложения 6 следует конкретный вид этой группы.

Таким образом, если отображение (6) переводит класс бент-функций в себя, то оно имеет вид

$$
g(x) \rightarrow g(A x+b)+\langle c, x\rangle+d .
$$

Напомним, что бент-функции, получающиеся одна из другой с помощью такого отображения называются аффинно эквивалентными. Из полученных результатов следует, что более общего подхода к эквивалентности бент-функций на основе изометричных отображений не существует.

\section{Список литературы}

1. Логачев О. А., Сальников А. А., Ященко В. В., Булевы функиии в теории кодирования и криптологии. МЦНМО, Москва, 2004.

2. Мак-Вильямс Ф. Дж., Слоэн Н. Дж. А., Теория кодов, исправляющих ошибки. Мир, Москва, 1979.

3. Токарева Н. Н., Бент-функции: результаты и приложения. Обзор работ. Прикладная дискретная математика (2009) 2, №1, 15-37.

4. Токарева Н. Н., Обобщения бент-функций. Обзор работ. Дискретный анализ и исследование операиий (2010) 17, №1, 34-64.

5. McFarland R. L., A family of difference sets in non-cyclic groups. J. Comb. Theory, Ser. A (1973) 15, 1-10.

6. Rothaus O., On bent functions. J. Comb. Theory, Ser. A (1976) 20, 300-305.

Статья поступила 19.04.2010.

Переработанный вариант поступил 14.05.2010. 